\title{
An Alternative Approach for Assessing Biogenicity
}

\author{
Joti Rouillard, ${ }^{1}$ Mark van Zuilen, ${ }^{2}$ Céline Pisapia, ${ }^{2}$ and Juan-Manuel Garcia-Ruiz ${ }^{1}$
}

\begin{abstract}
The search for signs of life in the ancient rock record, extreme terrestrial environments, and other planetary bodies requires a well-established, universal, and unambiguous test of biogenicity. This is notably true for cellular remnants of microbial life, since their relatively simple morphologies resemble various abiogenic microstructures that occur in nature. Although lists of qualitative biogenicity criteria have been devised, debates regarding the biogenicity of many ancient microfossils persist to this day. We propose here an alternative quantitative approach for assessing the biogenicity of putative microfossils. In this theoretical approach, different hypotheses-involving biology or not and depending on the geologic setting - are put forward to explain the observed objects. These hypotheses correspond to specific types of microstructures/systems. Using test samples, the morphology and/or chemistry of these systems are then characterized at the scale of populations. Morphologic parameters include, for example, circularity, aspect ratio, and solidity, while chemical parameters could include elementary ratios (e.g., N/C ratio), isotopic enrichments (e.g., $\delta 13 \mathrm{C}$ ), or chirality (e.g., molar proportion of stereoisomers), among others. Statistic trends distinguishing the different systems are then searched for empirically. The trends found are translated into "decision spaces" where the different systems are quantitatively discriminated and where the potential microfossil population can be located as a single point. This approach, which is formulated here on a theoretical level, will solve several problems associated with the classical qualitative criteria of biogenicity. Most importantly, it could be applied to reveal the existence of cellular life on other planets, for which characteristics of morphology and chemical composition are difficult to predict. Key Words: Biosignatures—Biogenicity_Astrobiology_Early life_Cellular life. Astrobiology 21, 151-164.
\end{abstract}

\section{Biosignatures and Biogenicity}

T RECENT DECADES, a large number of scientific studies have explored the boundaries of life, be they environmental [extreme settings (Jannasch, 1985; Belilla et al., 2019)], temporal [ancient rocks (Noffke et al., 2006; Sugitani et al., 2006)], or spatial [extraterrestrial environments (Oyama et al., 1976; Des Marais et al., 2008; Schwieterman et al., 2018)]. In these different contexts, the search for life relies on the detection of biosignatures, that is, features characteristic of life: living organisms, the evidence of life activity in its environment, or fossils of past organisms and traces of their past activity (Des Marais et al., 2008).

Defining a specific feature as a biosignature is far from straightforward. Neveu et al. (2018) proposed a list of instrumental and contextual criteria that must be satisfied in order for a feature to constitute a measurable convincing biosignature. These criteria are notably meant to avoid false negatives (i.e., misinterpreting a biosignature as an abiotic feature or a background noise) and false positives (i.e., misinterpreting an abiotic feature or a contamination as a biosignature).

According to Neveu et al. (2018), a good biosignature must first be generic: it should be a general feature shared by living organisms on Earth and potentially by other forms of life. Second, the environment where a biosignature is claimed to be found must be consistent with the (past) presence of life and the preservation of the biosignature. In other terms, the local habitability and preservability characteristics must be assessed (Westall et al., 2015).

The hypothesis of contamination must also be discarded. When searching for extraterrestrial biosignatures, the most critical source of contamination is terrestrial organic molecules and/or organisms, brought by spatial exploration itself (Kminek et al., 2014). In extreme terrestrial settings, discarding contamination means foremost proving the indigeneity of the biosignature (i.e., whether the feature has originated where it is measured/sampled, e.g., Santelli et al.,

\footnotetext{
${ }^{1}$ Laboratario de Estudios Cristalograficos, Instituto Andaluz de Ciencias de la Tierra, CSIC_-Universidad de Granada, Armilla, Spain.

${ }^{2}$ Institut de Physique du Globe de Paris, Université de Paris, CNRS UMR 7154, Paris, France.

(C) Joti Rouillard et al., 2020; Published by Mary Ann Liebert, Inc. This Open Access article is distributed under the terms of the Creative Commons License (http://creativecommons.org/licenses/by/4.0), which permits unrestricted use, distribution, and reproduction in any medium, provided the original work is properly credited.
} 
2010). For ancient life, beyond its indigeneity (Westall and Folk, 2003), the syngenicity of the biosignature-whether the feature originated at the same time as its host rock-must also be ensured to discard contamination (e.g., van Zuilen et al., 2007; Rasmussen et al., 2008; Javaux et al., 2010).

A good biosignature must also be reliable, it must be distinguishable from similar features produced abiotically. In other words, it must be possible to assess its biogenicity.

\section{Biogenicity: A Difficult Notion}

Numerous discussions have demonstrated the difficulty of rigorously assessing biogenicity (whether a feature has been formed by life or not). We focus here on examples from early life research (Schopf, 1983; García-Ruiz, 1994; Lowe, 1994; Brasier et al., 2002; van Zuilen et al., 2002; McLoughlin et al., 2010; Allwood et al., 2018; McMahon, 2019), but similar debates exist for astrobiology (Klein, 1978; McKay et al., 1996; Des Marais et al., 2008; Martel et al., 2012; Seager and Bains, 2015).

The reliability of most types of early life biosignatures has been debated to some extent. For example, isotopic signatures attributed to biological fractionation may also be obtained through abiotic processes (McCollom and Seewald, 2006). They do not constitute a good biosignature per se. Organic matter itself is not a good biosignature either, since it may be synthesized abiotically in many different environments (Chyba and Sagan, 1992; Lollar et al., 2002; García-Ruiz et al., 2003; Wolf and Toon, 2010; McCollom, 2013; Milesi et al., 2015; Ménez et al., 2018). Microbially influenced sedimentary structures (MISS), such as stromatolites, are a classical type of biosignature for early life (Hofmann et al., 1999; Tice and Lowe, 2004; Allwood et al., 2006; Nutman et al., 2016). Yet the biogenicity of some purported Archean MISS has been repeatedly questioned and has also been inferred to represent abiotic structures deposited under specific sedimentary conditions, features of fluid escape during diagenesis, or structures resulting from shear stress and/or compression (Lowe, 1994; Awramik and Grey, 2005; McLoughlin et al., 2008; Allwood et al., 2018; van Zuilen, 2018).

Some of the most heated controversies concerning biogenicity are focused on cellular remnants of early life. The structural simplicity of early prokaryotic life-forms (e.g., the absence of compartmentalization) prevents the use of comparative anatomy for phylogenetic affiliations, which is a fundamental aspect of Proterozoic and Phanerozoic paleontology. Microbial remnants in fossil assemblages have often been altered by early taphonomic events (e.g., Knoll and Golubic, 1979). Besides, localities where organic microfossils have been reported are metamorphosed and often affected by hydrothermal circulation (Rasmussen, 2000; Ueno et al., 2001; Brasier et al., 2002; Kiyokawa et al., 2006). Hydrothermal activity and metamorphism, by promoting the recrystallization of the mineral matrix and/or the circulation of organic fluids, can greatly modify the chemistry, ultrastructure, and even the general morphology of microfossils. Many abiotic objects, such as self-organized (organo)mineral aggregates (Hopkinson et al., 1998; García-Ruiz et al., 2003, 2017; Cosmidis and Templeton, 2016; Muscente et al., 2018; McMahon, 2019), mineral exfoliations (Wacey et al., 2015), tracks of the displacement of individual mineral grains [ambient inclusion trails; (e.g., Knoll and Barghoorn, 1974)], interstitial fillings (Brasier et al., 2005), or bitumen droplets (Buick, 1990), are consequently difficult to distinguish from true but structurally simple and strongly altered microfossils (Fig. 1).

Several of the early reported Archean microfossils were later reinterpreted as abiotic features [see review in Schopf (1983)], and some of the more recent claims of discovery of ancient microfossils-such as those occurring in the wellstudied Apex Chert, Pilbara, Western Australia, or in the Nuvvuagittuq Greenstone Belt, Quebec-are still extensively debated (Brasier et al., 2002; Schopf et al., 2002, 2018; García-Ruiz et al., 2003; Schopf and Kudryavtsev, 2009; Marshall et al., 2011; Wacey et al., 2015; Dodd et al., 2017; McMahon, 2019). These controversies regarding the nature of potential microfossils have particularly advanced our conceptual understanding of biogenicity.

\section{Assessing Biogenicity Using Biogenicity Criteria-Example of Microfossil Evaluation}

How can we assess the biogenicity of a specific feature found in the rock record? Is it possible to rigorously distinguish a biologic feature from its abiotic counterparts? These questions have been especially discussed for early life microfossils. In the following section, we briefly present the current protocols of biogenicity assessments for potential microfossils and illustrate them using two wellknown Archean assemblages. We then present the different issues associated with these protocols.

\subsection{Current protocols for assessing microfossil biogenicity}

Since the 1980s, several articles and book chapters have presented protocols for assessing the biogenicity of potential microfossils, in which the observed microstructures must verify a list of criteria to be considered true microfossils. The null hypothesis is nonbiogenicity, until proven otherwise (here, by satisfying a list of criteria), the object is considered abiotic. The choice of criteria and their number varies greatly between the different proposed lists.

In Table 1, we present the three main lists of criteria put forward by Buick (1990), Schopf et al. (2010), and Brasier and Wacey (2012). By way of illustration, the three lists of criteria are applied in Table 1 to two well-documented potential microfossil assemblages: one from the $1.88 \mathrm{Ga}$ Gunflint formation, whose biogenicity is widely recognized, and one from the $3.46 \mathrm{Ga}$ Apex chert, whose biogenicity is strongly debated. The individual criteria from the different lists are verified in the table by using conclusions drawn in the literature (Barghoorn and Tyler, 1965; Schopf, 1993; Brasier et al., 2002, 2005; Schopf et al., 2002, 2018; Schopf and Kudryavtsev, 2012; Lepot et al., 2017). The application of the different sets of criteria to the Gunflint and the Apex chert assemblages shows that:

(1) Evaluation of the biogenicity of the more recent, well-preserved Gunflint assemblage leads to the same result using the three lists of criteria-all the criteria are satisfied and biogenicity is proven.

(2) Evaluation of the biogenicity of the more ancient, hydrothermally affected Apex chert assemblage depends on the individual lists of criteria. When using the list from 

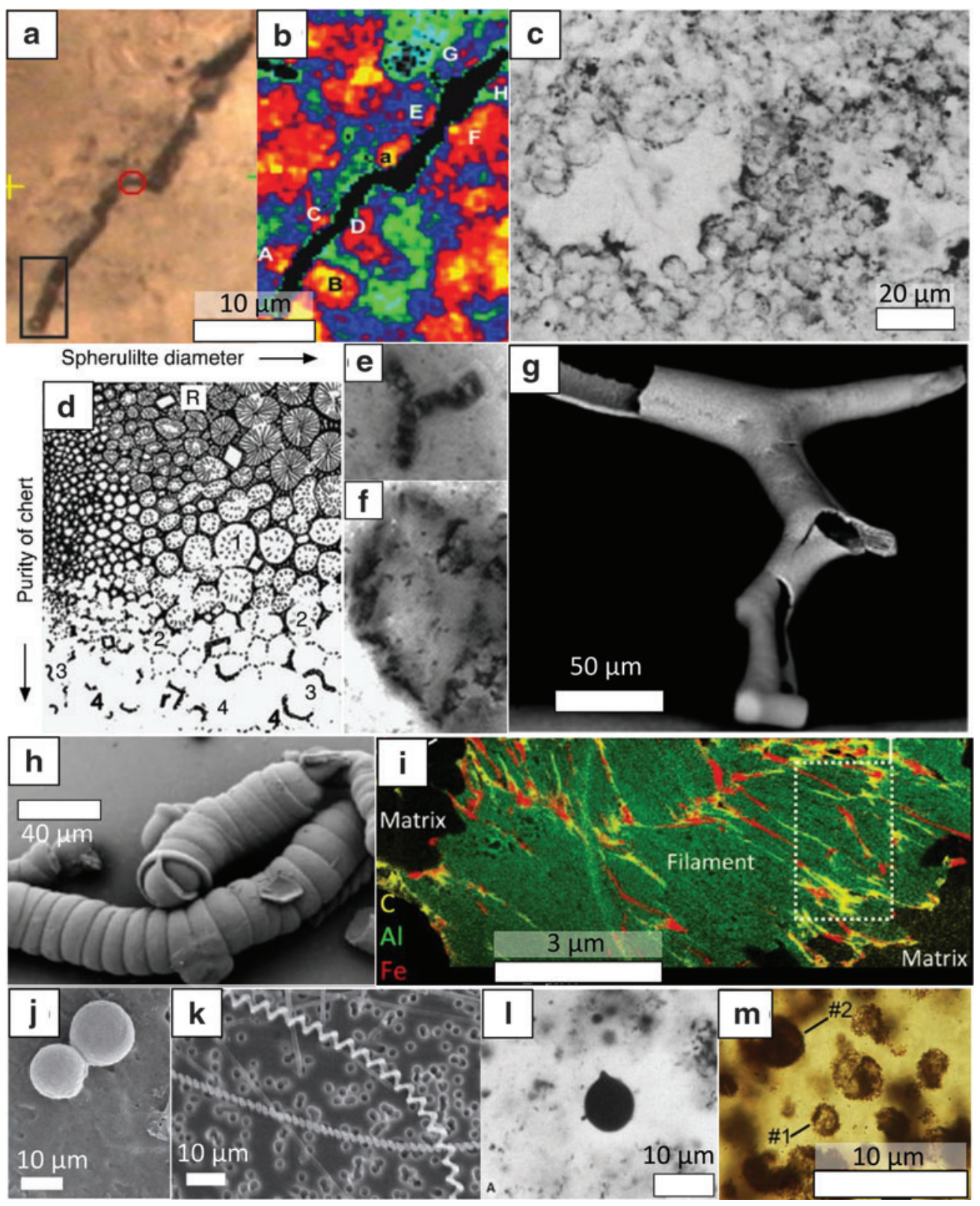

FIG. 1. Diversity of abiogenic microfossil-like objects. $(\mathbf{a}, \mathbf{b})$ Orientation of quartz crystals around a microstructure from the 3.46 Apex chert imaged by Raman mapping and indicating that this structure was formed by cracks in the mineral lattice after recrystallization of the mineral matrix (Bower et al., 2016). (c) Populations of siliceous spheroids clustered together and coated by organic material found in the Dresser formation (Buick, 1990). (d-f) Model (d) advanced to explain the formation of microstructures in the Apex chert (e,f) by the relocation of organic matter on the edges of growing silica spherulites (Brasier et al., 2005). (g) Bifurcating siliceous tube formed experimentally by chemical gardening, that is, immersion of metal salts into a highly alkaline ( $\mathrm{pH}>11)$ silica-rich solution (McMahon, 2019). (h) Filamentous silica/carbonate biomorphs synthesized experimentally by carbonate precipitation in an alkaline ( $\mathrm{pH}>9$ ) silica-rich medium (García-Ruiz et al., 2003). (i) Stacking of phyllosilicates imaged by elementary mapping in a filamentous microstructure from the Apex chert (Wacey et al., 2015). (j, k) Organomineral aggregates composed of carbon and sulfur and forming hollow spherical shells (j) and helical filaments (k) (Cosmidis and Templeton, 2016). (l) Organic microstructure from the Dresser formation and interpreted as a fossilized bitumen droplet (Buick, 1990). (m) Spheroidal hematitic aggregates from the 0.8 Chanda formation (Schopf et al., 2010). Color images are available online.

Buick (1990), the criterion of sedimentary setting is not satisfied - the assemblage originates from a paleohydrothermal vein-leading to a conclusion of nonbiogenicity. The two other lists do not lead to a clear conclusion on biogenicity, due to divergent views in literature. The verification of each of the biogenicity criteria from these two lists is controversial. Consequently, the scientific community still strives to reach a consensus regarding the origin of the Apex chert assemblage.

Where do these divergences in interpretation come from? How could the current protocols of biogenicity assessment be improved? These questions are discussed in the following paragraphs. 
Table 1. Summary of Different Lists of Biogenicity Criteria Proposed for the Evaluation of Microfossil Biogenicity and Verification IN THE Literature OF THE INDiVidual CRITERIA in Two Proposed Microfossil Assemblages

\begin{tabular}{lll}
\hline & & \multicolumn{2}{c}{ Applications } \\
\cline { 2 - 3 } List of criteria & Gunflint & Apex chert \\
\hline
\end{tabular}

Buick (1990)

(1) Objects embedded in the rock

(2) Sedimentary setting

(3) Objects above a minimal size

(4) Objects are organic

(5) Similar objects form a population

(6) Objects are hollow

(7) Objects display "cellular elaboration",
Yes (Barghoorn and Tyler, 1965)

Yes (Barghoorn and Tyler, 1965)

Yes (Barghoorn and Tyler, 1965)

Yes (Barghoorn and Tyler, 1965)

Yes (Barghoorn and Tyler, 1965)

Yes (Barghoorn and Tyler, 1965)

Yes (Barghoorn and Tyler, 1965)
Yes (Brasier et al., 2002; Schopf et al., 2002)

No (Brasier et al., 2002)

Yes (Schopf, 1993)

Yes (Brasier et al., 2002; Schopf et al., 2002)

Yes (Schopf, 1993)/No (Brasier et al., 2005)

Yes (Schopf et al., 2002)/No (Brasier et al., 2002)

Yes (Schopf et al., 2002)/No (Brasier et al., 2005)

Remarks Easy to apply in practice

Potential false negatives

Schopf et al. (2010)

(1) Species-specific, unimodal, narrow size distribution

(2) Different stages of development

(3) Limited range of morphologies

(4) Distinct cell walls

(5) Remnants of extracellular matrix

(6) Filaments: sinuosity dependent on length

(7) Benthic colonies: attachment to substrate
Yes (Barghoorn and Tyler, 1965)

Yes (Barghoorn and Tyler, 1965)

Yes (Barghoorn and Tyler, 1965)

Yes (Barghoorn and Tyler, 1965)

Yes (Barghoorn and Tyler, 1965)

-
Yes (Schopf, 1993)/No (Brasier et al., 2005)

Yes (Schopf, 1993)/No (Brasier et al., 2005)

Yes (Schopf and Kudryavtsev, 2012)/

No (Brasier et al., 2005)

Remarks Precise

Very specific to certain types of fossils

Brasier and Wacey (2012)

(1) Geologic context viable for life

(2) Morphology similar to modern unicellular life

(3) Evidences of biologic behavior

(4) Traces of biologic metabolism
Yes (Barghoorn and Tyler, 1965)

Yes (Barghoorn and Tyler, 1965)

Yes (Barghoorn and Tyler, 1965)

Yes (Lepot et al., 2017)
Yes (Schopf and Kudryavtsev, 2012)/

No (Brasier et al., 2005)

Yes (Schopf, 1993)/No (Brasier et al., 2005)

Yes (Schopf, 1993)/No (Schopf, 1993)

Yes (Schopf et al., 2018)/No (Brasier et al., 2005)

\section{Remarks Generalizable to other contexts \\ Criteria need to be précised}

Left column: summary of different lists of biogenicity criteria proposed for the evaluation of microfossil biogenicity. Right columns: verification in the literature of the individual criteria in two proposed microfossil assemblages: one widely recognized as biogenic (from the $1.88 \mathrm{Ga}$ Gunflint formation) and one whose biogenicity is very debated (from the $3.46 \mathrm{Ga}$ Apex chert).

Yes: verified; No: not verified; —: not discussed.

\subsection{Explaining divergences in interpretation using the current lists of criteria}

One reason for divergences in interpretation is that the biogenicity criteria in the existing lists are primarily qualitative. This may leave room for subjectivity, particularly for the criteria regarding cellular morphology or ecosystem behavior. The criterion of cellular hollowness, for example, can be interpreted in different ways, since it is not defined how thick a cell wall structure should be, relative to the overall cell dimensions. Brasier et al. (2002), therefore, described the Apex chert structures as solid organic aggregates, whereas Schopf and Kudryavtsev (2009) described them as clearly hollow. The need for quantification has consequently been repeatedly acknowledged in the literature. Brasier and Wacey (2012), for example, state that cellular morphology should be quantitatively distinguished from potential nonbiologic mimics. More recently, Chan et al. (2019) indicated the general need to build quantitative models of biologic and nonbiologic features. Neveu et al. (2018) also emphasized the "needs to quantify probabilities of the validity of abiotic null hypotheses." Assessing the probability of validity for different hypotheses could be much better achieved by using quantitative criteria. In general, defining quantitative criteria would probably facilitate discussions and improve the rigorousness of biogenicity evaluation. Yet very few studies have undertaken the actual quantitative comparison of microfossils and abiotic artifacts.

Another reason for these divergences in interpretation is that many of the defined criteria can lead to biased data selection. The criterion of biologic morphology appears to be convincingly verified for some of the Apex chert structures (Schopf, 1993); however, when looking at the entire population, many shapes are inconsistent with a biologic origin (Brasier et al., 2005). Choosing only a part of the data may evidently lead to interpretational biases. 


\subsection{Specific issues with the currently accepted lists of criteria}

The list proposed by Buick (1990), in particular, has proven to be useful over the years for assessing the biogenicity of many Proterozoic microstructures. However, as noted by Roger Buick in his original article, for the sake of safety, his set of criteria is selective. It could therefore exclude certain microstructures that are, in effect, true microfossils (false negatives). For example, if the microfossil has been strongly morphologically altered, the criterion of cellular elaboration may not be verified. Or, if the microfossil has lost most of its constituents during its evolution and is preserved by mineral texture alone, it would be discarded by the criterion of organic nature (Westall, 1999). Another example comprises the microfossils that are found within hydrothermal chimneys (e.g., remnants of chemolithotroph communities), which are excluded by the criterion of sedimentary setting. Considering that the early life fossil record is already sparse and looking for extraterrestrial life requires the investment of a great deal of resources, the potential elimination of true microfossils is problematic.

The set of criteria defined by Schopf et al. (2010) is precise, but also highly specific. It applies exclusively to coccoidal and filamentous microfossils. Although these morphologies represent the vast majority of reported Archean microfossil assemblages, other microfossil morphologies have also been reported, such as spindles (Sugitani et al., 2007, 2009). In general, the three different tests of biogenicity contain criteria that are mainly related to modern life on Earth $[e . g$., the criterion of cell-like morphospace as defined by Brasier and Wacey (2012)]. However, it is impossible to predict the types of morphologies that would be displayed by evolutionary distant organisms, that is, early life or extraterrestrial life.

The third list (Brasier and Wacey, 2012) has a broader purpose, since it is also designed for recognizing cellular life in extraterrestrial environments. In practice, however, the very general nature of the criteria $(e . g$. , the criterion of morphology relies on the comparison with a "cellular morphospace") may complicate their verification as long as they are not further specified.

\subsection{General issues with the current type of decision protocol}

In a general sense, there are also issues associated with this type of decision protocol. First, it does not give equal weight to biogenic and abiogenic explanations, since the null hypothesis is nonbiogenicity. In the context of life recognition, this is meant to increase certainty in the decision. Indeed, biogenicity is considered the most "extraordinary" claim and requires "extraordinary evidence" (Carl Sagan). Underlying this is an implicit probabilistic reasoning: the hypothesis of biogenicity is believed to have a lower probability of being true than the hypothesis of nonbiogenicity. However, two major caveats exist: (1) applying probabilities should rely on a minimal contextual knowledge. For both astrobiology and early life, the environmental context is poorly understood, and therefore applying probabilistic reasoning seems quite risky. (2) The probability that should be considered is not the probability of finding a microfossil in an extraterrestrial environment, or of finding a microfossil in an Archean rock. It is the probability that the specific object considered is a true cellular remnant, which is always higher: a potential microfossil is being evaluated because it has been selected as a promising candidate. This probability is also highly variable and will take a different value for each instance. In general, it is important to devise a decision protocol where no a priori assumption about the likeliness of alternative hypotheses is made.

Second, this type of protocol exclusively focuses the attention of the scientific community on cellular remnants. If the microstructure does not satisfy the biogenicity criteria, no information other than its "nonmicrofossil" nature is given. However, the main question that arises when observing a potential cellular biosignature is not whether it is an actual microfossil, but what its origin-whether biologic or nonbiologic-actually is. This is particularly important in the fields of early life and astrobiology, where the amount of data is limiting; any precise information can have important implications for our understanding of these (paleo)environments. It would therefore be important to devise a decision protocol that is nonbinary and gives a more precise interpretation of the nature of objects.

Since controversies regarding the biogenicity of some microfossil assemblages persist, the current protocols of biogenicity assessment appear to be insufficient in some cases. From the different issues raised in this section, we state that an ideal protocol of biogenicity assessment should encompass the following:

(1) Be quantitative.

(2) Use criteria that apply to an entire data set-in this context, the complete population of potential microfossils-to reduce the biases associated with data selection.

(3) Be applicable in various geologic records, including strongly degraded ones, to reduce the probability of false negatives.

(4) Use criteria that are as general as possible and may also be applicable to early/extraterrestrial life.

(5) Test various precise hypotheses regarding the nature of the object, with no a priori assumption about their relative likelihoods.

\section{Toward a New Protocol for Assessing Biogenicity}

With the technical improvements made in recent decades, an alternative method for assessing biogenicity can be envisioned. In this section, we present how lists of classically used biogenicity criteria could be replaced by quantitative decision spaces, thus solving most of the issues discussed above.

This new method for evaluating the biogenicity of potential microfossils is introduced here using the example of morphology measurements. Subsequently, we explain how this approach can be generalized for biogenicity assessments.

\subsection{The example of population morphometry}

As was explained in the previous section, the morphology of individual microorganisms may not be considered a robust biogenicity criterion (García-Ruiz, 1999; García-Ruiz et al., 2003; Cosmidis and Templeton, 2016; Muscente 
et al., 2018; McMahon, 2019). However, at the scale of entire microbial populations, life displays a specific quantifiable morphology. For populations of abiotic objects, the morphology is only controlled by environmental (in the broader sense) constraints. For biologic populations, supplementary types of constraints exist, which are due, in large part, to (1) physiology - notably the regulation of the cell cycle that affects both individual morphology and spatial relationships among cells (Collier and Shapiro, 2007; Wang and Levin, 2009; Young, 2010; Schumacher and SøgaardAndersen, 2017; Westfall and Levin, 2017) —and to (2) ecologic processes, which represent the ensemble of relationships (e.g., exchange of matter, of information, trophic relationships, sharing of resources) that exist between individuals in a biologic population or community (White, 1836; Warming, 1895; Phillips, 1931).

Darwinian evolution implies the existence of populations of biologic individuals, and it leads therefore necessarily to ecologic processes; even the simplest forms of life on modern Earth, and probably the first cellular organisms that appeared on Earth, display rudimentary forms of ecology (Hungate, 1966; Prosser et al., 2007; Egbert et al., 2011; Froese et al., 2014). It is a feature that is shared by, and specific to, life as we know it. When this is translated into a morphology specific to biologic populations, ecology would constitute a robust biosignature.

In one of our recent studies, we explored the potential of population morphometry for evaluating biogenicity (Rouillard et al., 2019). Morphometry is the quantitative study of morphology. Using morphometry, it is possible to locate objects on morphospaces, theoretical spaces in which axes represent continuous morphology-describing parameters (Raup, 1967). Morphometry has been used previously for studying (potential) Precambrian microfossil assemblages. Size distributions are the most frequent measurements and have been used to discuss issues of paleodiversity and/or biogenicity (e.g., Barghoorn and Tyler, 1965; Knoll and Golubic, 1979; Sugitani et al., 2013; Köhler and Heubeck, 2019). Measurements of tangent correlation lengths (Boal and $\mathrm{Ng}, 2010$ ) and oblateness (Sugitani et al., 2018) have also led to interesting paleobiologic and paleoevolutionary interpretations.

Rouillard et al. (2019) compared quantitatively the morphology of example modern communities of bacteria with the morphology of populations of two types of nonbiologic objects that could be mistaken for microfossils-interstitial spaces (e.g., Brasier et al., 2005) and self-assembled mineral aggregates grown in silica gel (e.g., García-Ruiz et al., 2003; Rouillard et al., 2018). Interstitial spaces, selfassembled mineral aggregates, and microbial communities are then defined as separate systems. Each system can be represented by multiple populations (e.g., the system of interstitial spaces can be represented by multiple images that each displays a different pore network in a rock). Sample images of these different systems, which correspond to different populations of objects/cells (Fig. $2 \mathrm{a}-\mathrm{c}$ ), were analyzed to extract parameters that describe the size and shape of individual objects and their general organization. Beyond the description of intrapopulation statistics (e.g., Fig. 2d), the different populations were compared quantitatively by using nondimensional descriptors of statistic distributions (mean/ standard deviation [SD], skewness, kurtosis-more details about the calculation of these parameters are presented in the notes at the end of this article).

This led to a system-level description of the different types of objects. It was found that the populations from the different systems exhibit different ranges for distribution descriptors (e.g., Fig. 2e). In addition, specific correlations between population-describing parameters could be observed (Fig. 2e). Depending on the nature of items compared, distinct "levels of study" can be defined, for example, the object, the population, or multiple populations. A level of study $\mathrm{A}$ is higher than a level of study B if it considers individually items $\mathrm{A} 1, \ldots$ An, which are each composed of the items considered individually in B (i.e., A1 contains B1, $\ldots, \mathrm{Bn})$. A critical result of this study is that the distinction between the systems improves with the level of study: from the level of a single object, to the level of a population of objects, and finally to the level of multiple populations.

\subsection{From discriminant analyses to decision spaces}

The morphometric characterization of populations, as described in the previous paragraph, shows that biogenicity can be evaluated by using quantitative methods. A first possible method is the use of discriminant analyses (Lachenbruch and Goldstein, 1979; McLachlan, 2004; Bishop, 2006). By applying automated discriminant analyses on the distribution descriptors from all sample populations, it was found that a good separation of the different systems (interstitial spaces, biomorphs, and microbial cells) is possible (Fig. 3a). However, an important remark must be made. To be of interest to biogenicity assessment, discrimination should be valid for any population from the different systems. However, the results of automated discriminant analyses are strongly dependent on data input (Fig. 3b-d); they find the linear combination of parameters that, at best, separate the given ensemble of populations. If the sample populations used in the study do not encompass the heterogeneity of the systems, the results cannot be used to assess biogenicity in a general context.

One way to solve this issue could be to integrate a larger amount of sample populations from more sources. This would significantly improve the description of the systems, and the applicability of the results of the discriminant analyses would be increased. However, the difficulty of accessing a large amount of data in natural contexts, and the fact that such results may not be applicable to unknown contexts (e.g., extraterrestrial), means that this is not an ideal solution.

Alternatively, to obtain a more robust discrimination (less dependent on data input), we propose considering only specific parameters. The critical question concerns the choice of these parameters. These parameters should translate the specificities of life-features shared by all and only lifeinto a range of numeric values that distinguish the biologic system from the abiotic systems. The study of statistic morphometry hints at a promising answer. As stated earlier, it was found that the morphologic distinction between the different systems improves with the level of study (see end of Section 5.1). Single objects from the different systems cannot be reliably distinguished. At the level of populations, the different systems appear to have different types of control on shape (Fig. 2d). At the multipopulation level, the variability of statistic distributions distinguishes the 

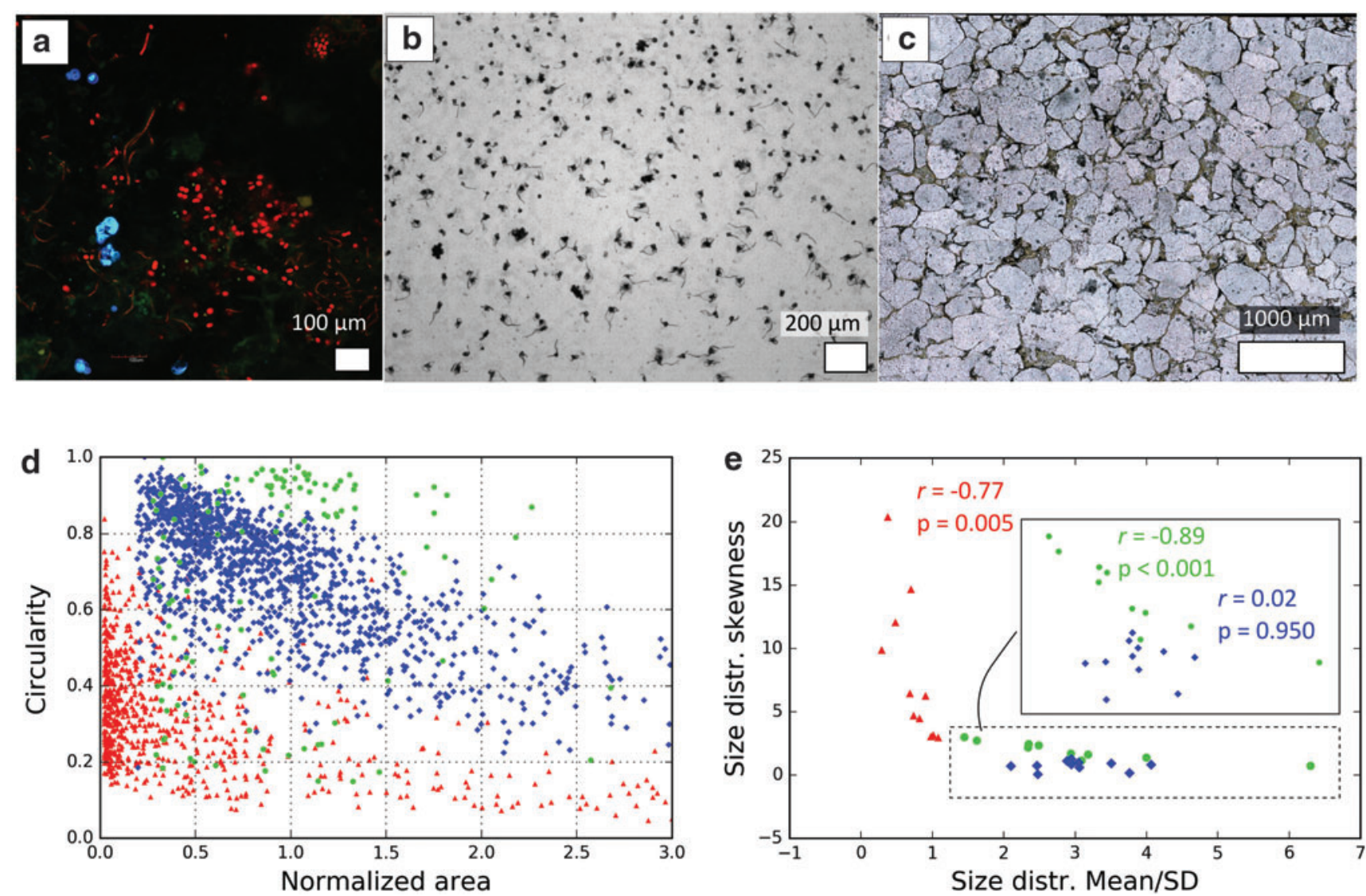

FIG. 2. (a-c) Example populations from each of the three systems (natural microbial communities, biomorphs, and interstitial spaces in clastic rocks) compared in Rouillard et al. (2019). (a) Stromatolite-dwelling microbial community from Alchichica lake (Mexico). Picture taken using Confocal Laser Scanning Microscopy without staining. (b) Silica-witherite biomorphs grown in silica gel. (c) Interstitial spaces between quartz clasts in a sandstone filled by a dark ferruginous cement. (d) Relationship between area (normalized to the mean of the distribution) and circularity in the three different systems: a population of interstitial spaces (red triangles), a population of biomorphs (blue diamonds), and a microbial community (green circles). Each point represents a single particle (interstitial space, biomorph, or cellular organism). (e) Mean/SD of size distributions plotted against the skewness of size distribution in different populations from the same systems. The inset is a zoom-in of the area framed by the dotted line. Each point represents a population. The Pearson correlation coefficient $r$ between the shapes of the different distributions and the associated probability of noncorrelation $p$ are given for each system. Details about the calculation of $r$ and $p$ are given in the notes at the end of this article. Note the different types of correlation distinguishing the three systems. All data from Rouillard et al. (2019). SD, standard deviation. Color images are available online.

different systems very consistently (Fig. 2e). We propose the hypothesis that statistic trends occurring at higher levels are due to more general processes, and that they are also more likely to be shared by all life. From this study, the degree of correlation between distribution-describing dimensions, or the slope of the linear fit of these correlations (Fig. 2e), for example, represents promising quantitative parameters for evaluating biogenicity. The origin of these differences in trends is not understood yet; it is probable that they are due to processes specific to life, such as ecology. The choice of the discriminating parameters relies here on a purely empiric analysis, making it a data-driven procedure, less prone to the human errors that could occur during data interpretation.

By using such parameters, it is therefore possible to devise decision spaces specific to the systems considered (example shown in Fig. 4, using the data shown in Fig. 2e). Decision spaces are parametric spaces in which one sample (the potential biosignature) is represented by a single point and the location of the sample enables one to make a decision regarding its nature. A “decision space," we propose here, may rely on different types of measurements (not only measurements of morphology). For instance, some parameters may reflect trends found in individual measurements of elementary/molecular composition, of structural order, of isotopic enrichments, and so on. Decision spaces can therefore integrate an array of various information quantitatively. The number of dimensions of the space corresponds to the final number of selected parameters. In these spaces, the systems occupy defined volumes (in the wider sense) with a minimum overlap. If the data analysis has been correctly conducted, since the trends evidenced are shared at the scale of the system, such a discrimination is more robust to data input than automated discriminant analysis. Also, since it is easier to reason with single parameters than linear combinations of many parameters, the discussion of the specific parameters to be considered for the decision is 

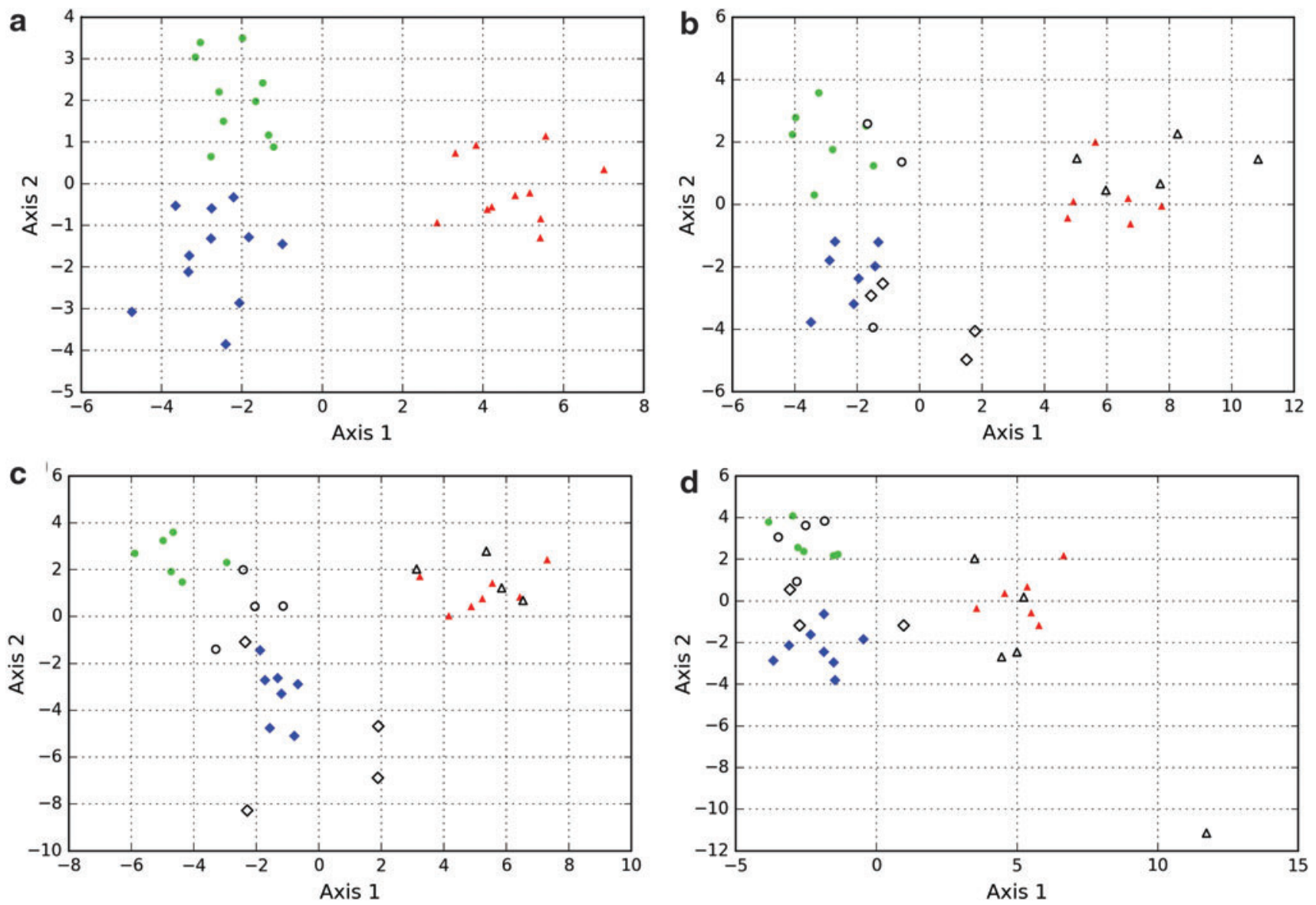

FIG. 3. Discriminant analyses conducted on populations of interstitial spaces, biomorphs, and microorganisms. The parameters used are the different adimensional distribution descriptors (mean/SD, skewness, and kurtosis) of the statistic distributions of size, circularity, and solidity_nine parameters in total. The axes correspond to linear combinations of all parameters that allow the maximization of the variance between the different systems in a two-dimensional space (Axis-1 vs. Axis-2). (a) Discriminant analysis run with the complete set of populations available from the three systems. (b-d) Discriminant analyses run with only 20 populations chosen randomly out of the 32 populations available. These populations constitute a training set, shown as filled symbols. The remaining populations - the test set—are shown as hollow symbols on the obtained discriminating spaces. Note that the results of the discriminant analyses are variable depending on the training set. The discrimination achieved is imperfect, since some populations of the test sets plot away from their system of origin. This indicates that the training sets do not encompass the heterogeneity of the systems. Data used to make the analyses are from Rouillard et al. (2019). Color images are available online.

easier than the discussion of the principal components obtained using discriminant analyses.

In the illustrative example of decision space shown in Fig. 4, the three systems are represented by the three groups of populations characterized in the work of Rouillard et al. (2019) (filled green circle, filled red triangle, and filled blue diamond). The three systems are discriminated according to the relationship between the skewness and the mean/SD of the size distributions in the system's populations (Fig. 2e), with the linear correlation coefficient between the two parameters plotted on the $y$-axis and the slope of the linear fit plotted on the $x$-axis. The three systems are correctly separated in this space. Assuming that enigmatic microstructures are discovered in a new sample, according to their geologic context, the following three hypotheses"interstitial spaces," "silica/carbonate biomorphs," and "microbial community" — are put forward to explain their origin. Depending on the correlation it exhibits, the group of populations of microstructures in this sample can be located alongside these systems in the space shown in Fig. 4 (see star in Fig. 4), and it may be attributed to the closest system (interstitial spaces in this example). We note that in this specific decision space, due to the small number of populations considered in the work of Rouillard et al. (2019), the data available as of now for each system are plotted as one (filled) symbol, which means that it is not possible to give numeric probabilities for the sample to belong to each of the defined hypotheses/ systems. By characterizing distinct groups of populations from these systems (coming, e.g., from distinct natural locations/ experimental settings), each system could be represented by a cloud of points (see hollow symbols in Fig. 4), thus enabling the application of statistic models to determine the probabilities of validity for each hypothesis/system.

In the following section, we expand from this example to explain how this approach can be generalized and improved for the biogenicity assessment of various biosignatures. 


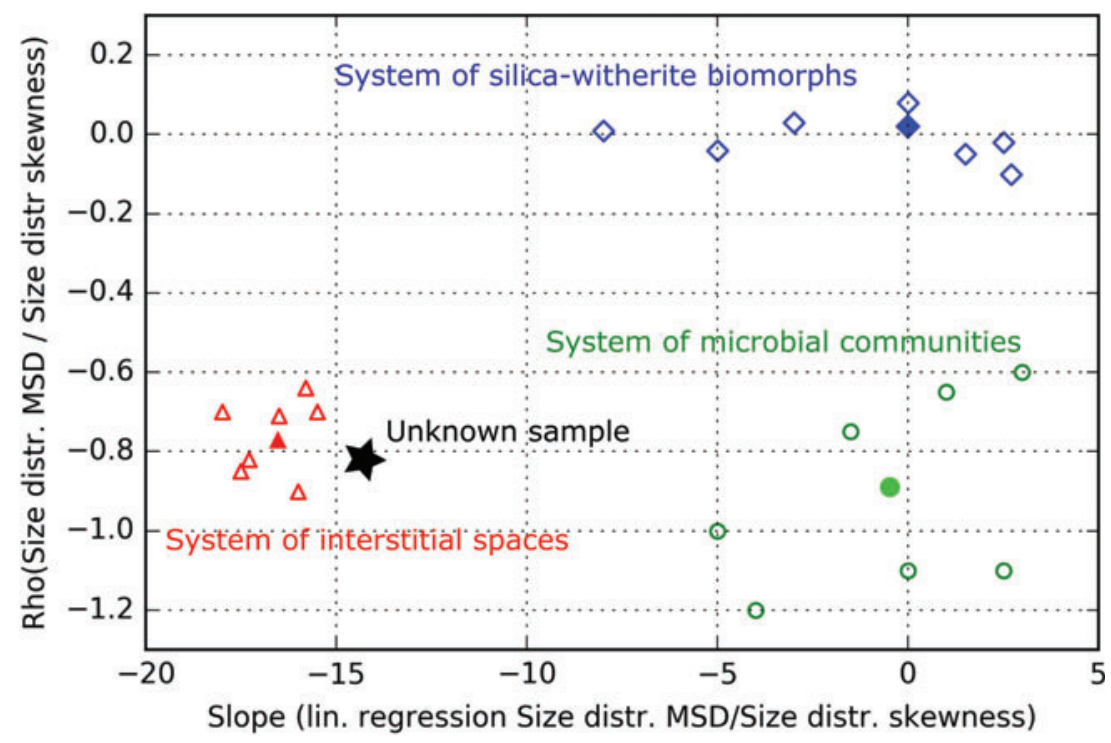

FIG. 4. Example of a multipopulation-level decision space devised using data from Rouillard et al. (2019) (filled symbols; parameters plotted on Fig. 2e) and hypothetical groups of populations (hollow symbols). Values on the $y$-axis correspond to the Pearson correlation coefficient between the mean/SD and skewness of size distributions measured for the populations in the three different systems (they correspond to the $r$ values given in Fig. 2e). Values on the $x$-axis represent the slopes of the linear fits between the same parameters in the three systems. The filled green circle, the filled red triangle, and the filled blue diamond therefore characterize the statistic trend found, respectively, within the group of 10 microbial communities, the group of 11 populations of interstitial spaces, and the group of 11 populations of silica-witherite biomorphs shown in Fig. 2e. Note that no statistically significant linear correlation was found between these two parameters for the 11 populations of silica-witherite biomorph populations (this could come from the overlap of distinct growth conditions in gels). A hypothetical group of populations of microstructures of unknown nature, represented by a star, may be located along these three systems in the decision space. By considering supplementary hypothetical groups of populations (hollow symbols), systems may be represented by a set of points. Numeric probabilities that the sample belongs to each of the different systems can then be given. Note that it is possible to discriminate any pair among the three systems using only one parameter, but the two parameters are required to discriminate all three systems. Color images are available online.

\section{A General Protocol for Biogenicity Assessments Integrating Decision Spaces}

5.1. When evaluating the biogenicity of a proposed biosignature, the different explanatory hypotheses should first be defined. Hypotheses depend on the geologic context, that is, what types of objects/features could be expected in such an environment? Hypotheses may involve biology or not, and to different degrees, integrating a potentially more nuanced view of biogenicity.

To illustrate this, we consider the hypothesis of the discovery of filamentous microstructures in a paleohydrothermal chimney, which is similar to structures reported in the work of Schopf (1993) and Dodd et al. (2017). Based on current knowledge of hydrothermal systems, a specific set of hypotheses can be made regarding the nature of these structures. They may represent (a) fossils of chemolithoautotrophs living in situ (Jannasch, 1985; Schuler et al., 2017; Ward et al., 2017); (b) dead organisms fallen from the water column and circulated by hydrothermal fluids (Duda et al., 2018); (c) mineral stalks produced by the metabolism of bacteria; (d) mineral gardens grown due to chemical gradients between hydrothermal fluids and seawater (Hopkinson et al., 1998; McMahon, 2019; Johannessen et al., 2020); (e) biomorphic, hybrid mineral aggregates growing in hydrothermal fluids (García-Ruiz et al., 2003, 2017); or (f) fillings in interstitial spaces in the mineral lattice by organicrich fluids (Buick, 1990; Brasier et al., 2005). The pa- leoenvironmental interpretation is important at this stage. The hypotheses formulated for structures found in a hydrothermal chimney are distinct from the hypotheses formulated for similar structures found, for example, in a sedimentary rock affected by later hydrothermal events.

We emphasize that all hypotheses have the same position in the protocol; there is no null hypothesis and no a priori assumption of their different respective probabilities. As such, we claim that this method follows precisely the recommendation made by Martin Brasier, that is, "that no claim for life can be accepted until all alternative abiotic explanations for its formation are discarded." The selection of the hypotheses is critical, since reducing their number improves the quality of discrimination (fewer parameters are required to separate fewer systems, see caption of Fig. 4), but increases the risk of missing the valid hypothesis.

5.2. The systems (corresponding to the different hypotheses) should then be characterized exhaustively, by using test samples, along with the proposed biosignature. The goal is to characterize systems as they are at the end of the geologic history of the proposed biosignature, that is, at the time of sampling of the proposed biosignature (Fig. 5). This requires a good knowledge of local geologic history [evolution of pressure, temperature, and events of fluid circulation (Chan et al., 2019), including taphonomic effects (Knoll and Golubic, 1979)] and the way this specific history would affect the candidate systems. 
This knowledge may be acquired by tracing the systems in the well-known geologic record (Jones et al., 2001; Guidry and Chafetz, 2003; Konhauser et al., 2004; Campbell et al., 2015) or by using artificial taphonomy/diagenesis/alteration experiments (Oehler, 1976; Picard et al., 2015; Alleon et al., 2016a, 2016b). For geologic records affected by hydrothermalism, it is crucial to know at which time of geologic history hydrothermal circulation occurred; the effects of hydrothermal fluids on nonfossilized bacteria would presumably differ from the effects of a late hydrothermal circulation on already fossilized bacteria. Although the specific example of morphologic measurements was put forward in the previous section, other types of measurements (e.g., chemical maps) would improve the quality of characterization and the reliability of subsequent discrimination.

The issue of sampling is of paramount importance. To obtain statistically significant results, and also to prevent biased data selection and gerrymandering, the entire proposed biosignature should be characterized. For example, in the case of a putative biofilm with cellular remnants that is under study, all of the biofilm should be characterized. Moreover, since it is critical to search for and test statistic trends that exist at the multipopulation level, several populations of objects of a system must be measured. If the proposed biosignature occurs only in one sample, this obstacle may be overcome by spatially dividing the volume of the sample in a systematic way, thus generating several spatially segregated populations of objects. The test samples from the different systems should be characterized in a similar way, at the same scale (including spatial division of the samples) and with the same precision, so that subsequent statistical analyses can be compared.

5.3. Data obtained from the test samples are explored statistically to empirically find higher level trends (observable only at the multipopulation level) that distinguish the systems that are being studied. Specific parameters are selected that translate these distinct trends into separate numeric ranges (see parameters used in Fig. 4). Such trends may reflect classical, qualitative biogenicity criteria in a quantitative manner. For example, higher level trends found by using statistic morphometry may be linked to the criteria of "biologic morphology" and "biologic behavior." Similar trends found through other types of quantitative measurements [e.g., spatial patterns of chemicals or molecular weight distributions, see Dorn et al. (2011)] could reflect other classical biogenicity criteria $[e . g$. , the criteria of "biologic metabolism," see Brasier and Wacey (2012); Kaneko and Furusawa (2018)]. The choice of the parameters depends on the geologic history (Fig. 5); the parameters chosen for the inferred geologic history (e.g., Parameters 1 and 2 in Fig. 5, panel 2) may be less efficient at discriminating the different systems than other parameters if the systems were not degraded at all (compare the two graphs in panel 1) or if they underwent a different geologic history (compare the two graphs in panel $2^{\prime}$ ).

5.4. A multiparametric decision space can be built from the selected parameters, integrating potentially different types of measurements. It is clear from the preceding considerations that this decision space is not a general space that may be applied indiscriminately to any proposed biosignature. The decision space is relative to a certain type of biosignature and to a certain geologic context (Fig. 5). The nature and biogenicity (and potentially contaminant nature) of the proposed biosignature may be evaluated by simply locating its position in the decision space (stars in Fig. 5, panel 2). Such a space also allows the application of statistical models. Consequently, it is possible to calculate probabilities of validity for the different hypotheses, according to the different parameters of the decision space. It is theoretically possible that no reliable decision can be reached (e.g., stars S2 and S3 in Fig. 5, panel 2). This may be due either to an insufficient discrimination between systems (star S2) or to missing the valid hypothesis (star S3).

5.5. Since decision spaces are related to a certain geologic context, beyond the nature of the proposed biosignature they can also help in assessing potential contamination issues by giving information pertaining to the syngenicity and indigeneity of the proposed biosignature. Indeed, if the feature did not originate at the same time or at the same place as the host rock, there is a high probability that it will have undergone a different geologic history; its final characteristics (assuming the characteristics of the feature are dependent on its geologic history) differ from a corresponding syngenetic and indigenous feature (compare the location of green ellipses in Fig. 5, panels 1, 2, and 2', parameters 1 and 2). However, decision spaces should be used with caution in the context of contamination assessment, since there is a danger that a contaminant feature might overlap with one of the systems in the decision space. Contaminant systems could of course also be integrated as separated hypotheses into decision spaces, but this matter is beyond the scope of the current article.

\section{Concluding Remarks}

The protocol proposed here for assessing biogenicity represents a promising way to solve most of the issues associated with a classical protocol that relies on a list of biogenicity criteria. A number of alternative hypotheses may be defined, involving biology or not. There is no null hypothesis and no a priori assumption about their respective probabilities. Beyond the information pertaining to biogenicity, the use of such a protocol-depending on the specificity of hypotheses confronted-can also give precise information about the nature of the enigmatic feature that is put forward as a potential biosignature. In the context of contamination assessment, it can also help (although with a cautionary note) in evaluating the syngenicity and indigeneity of a feature. The discrimination relies on distinct trends observable only at large statistical scales, trends that may be due to general processes shared by all life. As a consequence, the possibility of biased data selection is reduced, and the protocol may be generalizable to different types of life for which absolute sizes, shapes, or chemical concentrations are unpredictable. Instead of checking qualitative, often binary criteria, this method compares the values taken by relevant biologic and abiotic systems for specific parameters; this will hopefully help the discussions and reduce subjectivity. However, the protocol proposed here requires an extensive knowledge of natural systems. Right now, decision spaces can only be built using a limited number of samples and limited types of measurements. In 


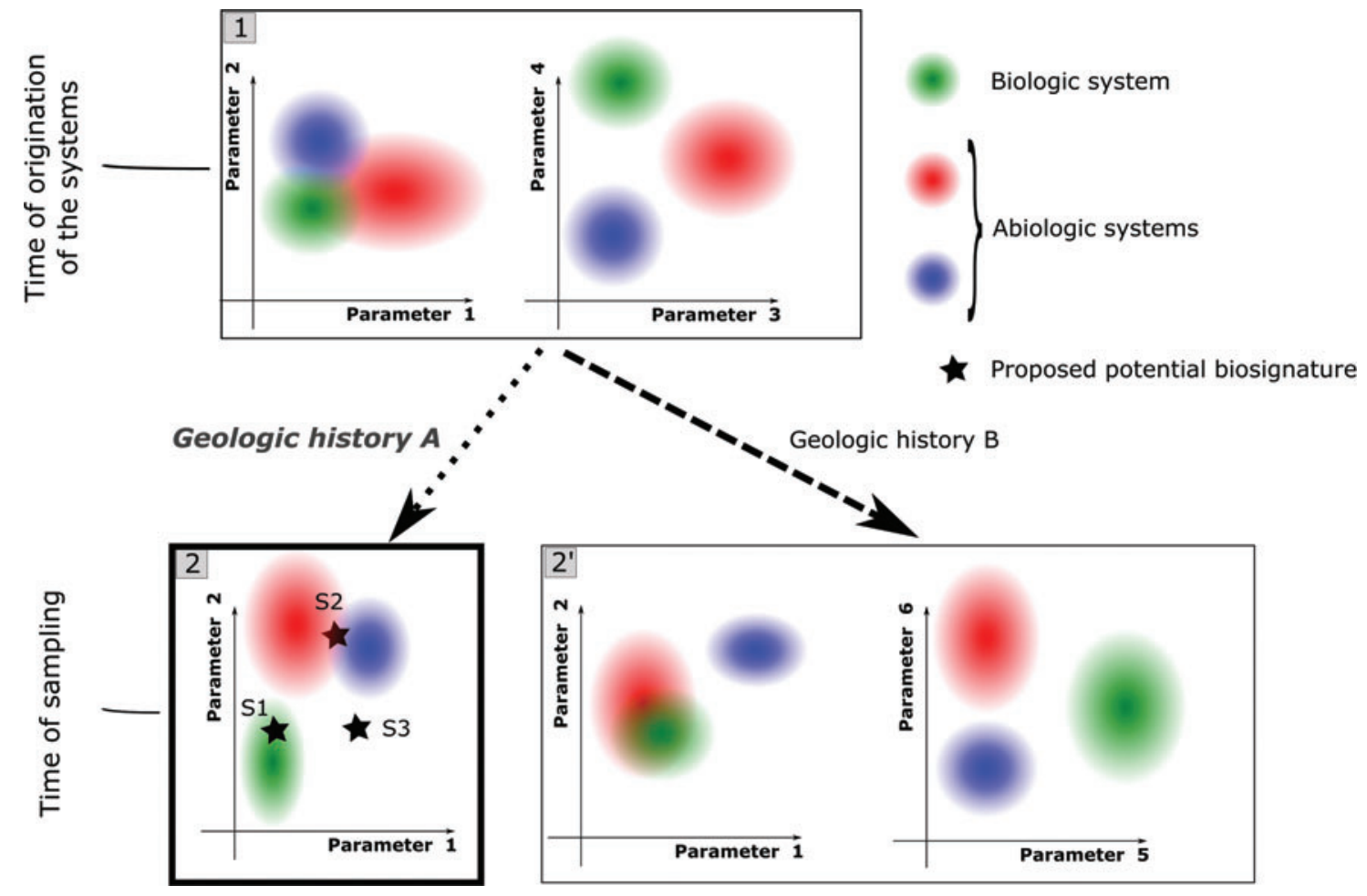

FIG. 5. Illustration of the importance of parameter choice for assessing biogenicity using decision spaces. Here one example of a biologic system and two examples of abiotic systems are shown, located, respectively, by green, blue, and red ellipses in spaces built with couples of parameters. (1) Shows the systems before geologic evolution, while (2, 2') show the systems altered by geologic history (pressure, temperature, fluid circulations). The two dotted arrows indicate two different geologic histories. Depending on the history and on the parameters considered, the initial characteristics of the different systems can undergo various changes. For example, with respect to parameters 1 and 2, systems may diverge [green and blue systems from (1) to (2)] or converge [green and red systems from (1) to (2'). Parameters chosen for biogenicity assessment are those that maximize discrimination between the systems at the end of the geologic history of the proposed biosignature. Potential biosignatures are located by stars in (2). The geologic history inferred for these proposed biosignatures is A. The parameters chosen to build the decision space are consequently 1 and 2 . Here, S1 may be interpreted as biogenic. S2 is interpreted as abiotic, but no information regarding its nature (blue system or red system) can be obtained. S3 is too far from any of the three systems, and no decision about its biogenicity should be made using this decision space. Statistic models may be applied to give actual probabilities of validity for the different systems. Color images are available online.

the future, results obtained from similar measurements on various samples from different systems should be gathered in online databases. The reliability and applicability of the protocol would thus be drastically increased.

\section{Notes}

(1) The mean divided by SD (mean/SD), which describes the relative width of the distribution, is calculated according to the following:

$$
M / S D(X)=\frac{\mu(X)}{\sigma(X)}
$$

(2) Skewness, which describes the asymmetry of the distribution, is calculated according to the following:

$$
S(X)=\frac{1}{G} \sum_{i=1}^{G}\left(X_{i}-\mu(X) / \sigma(X)\right)^{3}
$$

(3) Kurtosis, which describes the importance of tails in the distribution, is calculated according to the following:

$$
K(X)=\frac{1}{G} \sum_{i=1}^{G}\left(X_{i}-\mu(X) / \sigma(X)\right)^{4}
$$

The linear correlation coefficient $r$ between two parameters $X$ and $Y$, measured on a population of $G$ points, is calculated according to the following:

$$
r(X, Y)=\frac{\sum_{i=1}^{G}\left(X_{i}-\mu(X)\right)\left(Y_{i}-\mu(Y)\right)}{\sigma(X) \sigma(Y)}
$$

The significance of the correlation $p$ (or probability that the two parameters are not correlated) varies with the number of points $G$ and is calculated with a two-tailed test according to the following:

$$
p(X, Y)=r(X, Y) \sqrt{\frac{G-2}{1-r(X, Y)^{2}}}
$$

No issue related to ethics exist for the research presented in this article. 


\section{Acknowledgments}

J.-M.G.-R. acknowledges the Ministerio de Economia y competividad of Spain for funding the project CLG201678971-P. The authors thank two anonymous reviewers and the editors at astrobiology for their useful comments that helped improving a previous version of the article. The authors also thank J.D. Hayes for careful copy editing of the article.

\section{Author Disclosure Statement}

No competing financial interests exist.

\section{Funding Information}

This project has received funding from the European Research Council, under the European Union's Horizon 2020 research and innovation programme (grant agreement no. 694894) and the Seventh Framework Programme-FP7/ 2007-2013 (grant agreement no. 340863).

\section{References}

Alleon J, Bernard S, Le Guillou C, et al. (2016a) Early entombment within silica minimizes the molecular degradation of microorganisms during advanced diagenesis. Chem Geol 437:98-108.

Alleon J, Bernard S, Le Guillou C, et al. (2016b) Molecular preservation of $1.88 \mathrm{Ga}$ gunflint organic microfossils as a function of temperature and mineralogy. Nat Commun 7: 11977.

Allwood AC, Walter MR, Kamber BS, et al. (2006) Stromatolite reef from the early Archaean era of Australia. Nature 441:714-718.

Allwood AC, Rosing MT, Flannery DT, et al. (2018) Reassessing evidence of life in 3,700-million-year-old rocks of Greenland. Nature 563:241-244.

Awramik SM and Grey K (2005) Stromatolites: biogenicity, biosignatures, and bioconfusion. In Presented at the Optics and Photonics 2005, edited by RB Hoover, GV Levin, AY Rozanov, and GR Gladstone. San Diego, CA, p 59060.

Barghoorn ES and Tyler SA (1965) Microorganisms from the Gunflint chert. Science 147:563-577.

Belilla J, Moreira D, Jardillier L, et al. (2019) Hyperdiverse archaea near life limits at the polyextreme geothermal Dallol area. Nat Ecol Evol 3:1552-1561.

Bishop CM (2006) Pattern Recognition and Machine Learning. Information Science and Statistics, Springer, New York.

Boal D and Ng R (2010) Shape analysis of filamentous Precambrian microfossils and modern cyanobacteria. Paleobiology 36:555-572.

Brasier MD and Wacey D (2012) Fossils and astrobiology: new protocols for cell evolution in deep time. Int J Astrobiol 11: 217-228.

Brasier MD, Green OR, Jephcoat AP, et al. (2002) Questioning the evidence for Earth's oldest fossils. Nature 416:76-81.

Brasier MD, Green OR, Lindsay JF, et al. (2005) Critical testing of Earth's oldest putative fossil assemblage from the $\sim 3.5$ $\mathrm{Ga}$ apex chert, Chinaman Creek, Western Australia. Precambrian Res 140:55-102.

Buick R (1990) Microfossil recognition in Archean rocks: an appraisal of spheroids and filaments from a 3500 m.y. old chert-barite unit at North Pole, Western Australia. Palaios 5: 441-459.
Campbell KA, Lynne BY, Handley KM, et al. (2015) Tracing biosignature preservation of geothermally silicified microbial textures into the geological record. Astrobiology 15:858-882.

Chan MA, Hinman NW, Potter-McIntyre SL, et al. (2019) Deciphering biosignatures in planetary contexts. Astrobiology 19:1075-1102.

Chyba CF and Sagan C (1992) Endogenous production, exogenous delivery and impact-shock synthesis of organic molecules: an inventory for the origins of life. Nature 355: $125-132$.

Collier J and Shapiro L (2007) Spatial complexity and control of a bacterial cell cycle. Curr Opin Biotechnol 18:333-340.

Cosmidis J and Templeton AS (2016) Self-assembly of biomorphic carbon/sulfur microstructures in sulfidic environments. Nat Commun 7:12812.

Des Marais DJ, Nuth JA, Allamandola LJ, et al. (2008) The NASA astrobiology roadmap. Astrobiology 8:715-730.

Dodd MS, Papineau D, Grenne T, et al. (2017) Evidence for early life in Earth's oldest hydrothermal vent precipitates. Nature 543:60-64.

Dorn ED, Nealson KH, and Adami C. (2011) Monomer abundance distribution patterns as a universal biosignature: examples from terrestrial and digital life. J Mol Evol 72: 283-295.

Duda J-P, Thiel V, Bauersachs T, et al. (2018) Ideas and perspectives: hydrothermally driven redistribution and sequestration of early Archaean biomass - the "hydrothermal pump hypothesis." Biogeosciences 15:1535-1548.

Egbert MD, Barandiaran XE, and Di Paolo EA. (2011) Behavioral metabolution: the adaptive and evolutionary potential of metabolism-based chemotaxis. Artif Life 18:1-25.

Froese T, Virgo N, and Ikegami T (2014) Motility at the origin of life: its characterization and a model. Artif Life 20:55-76.

García-Ruiz JM (1994) Inorganic self-organisation in Precambrian cherts. Origin Life Evol Biosphere 24:451-467.

García-Ruiz JM (1999) Morphological behavior of inorganic precipitation systems. In Instruments, Methods, and Missions for Astrobiology II, Proceedings of SPIE. Denver, pp 74-82.

García-Ruiz JM, Hyde ST, Carnerup AM, et al. (2003) Selfassembled silica-carbonate structures and detection of ancient microfossils. Science 302:1194-1197.

García-Ruiz JM, Nakouzi E, Kotopoulou E, et al. (2017) Biomimetic mineral self-organization from silica-rich spring waters. Sci Adv 3:e1602285.

Guidry SA and Chafetz HS (2003) Depositional facies and diagenetic alteration in a relict siliceous hot-spring accumulation: examples from Yellowstone National Park, U.S.A. J Sediment Res 73:806-823.

Hofmann HJ, Grey K, Hickman AH, et al. (1999) Origin of 3.45 Ga coniform stromatolites in Warrawoona Group, Western Australia. Geol Soc Am Bull 111:1256-1262.

Hopkinson L, Roberts S, Herrington R, et al. (1998) Selforganization of submarine hydrothermal siliceous deposits: evidence from the TAG hydrothermal mound, $26^{\circ} \mathrm{N}$ MidAtlantic Ridge. Geology 26:347-350.

Hungate R (1966) The Rumen and Its Microbes, Academic Press. New York.

Jannasch HW (1985) Review lecture-the chemosynthetic support of life and the microbial diversity at deep-sea hydrothermal vents. Proc $R$ Soc Lond B Biol Sci 225: 277-297.

Javaux EJ, Marshall CP, and Bekker A (2010) Organic-walled microfossils in 3.2-billion-year-old shallow-marine siliciclastic deposits. Nature 463:934-938. 
Johannessen KC, McLoughlin N, Vullum PE, et al. (2020) On the biogenicity of Fe-oxyhydroxide filaments in silicified low-temperature hydrothermal deposits: implications for the identification of $\mathrm{Fe}$-oxidizing bacteria in the rock record. Geobiology 18:31-53.

Jones B, Renaut RW, and Rosen MR (2001) Taphonomy of silicified filamentous microbes in modern geothermal sintersimplications for identification. Palaios 16:580-592.

Kaneko K and Furusawa C (2018) Macroscopic theory for evolving biological systems akin to thermodynamics. Annu Rev Biophys 47:273-290.

Kiyokawa S, Ito T, Ikehara M, et al. (2006) Middle Archean volcano-hydrothermal sequence: bacterial microfossilbearing 3.2 Ga Dixon Island Formation, coastal Pilbara terrane, Australia. Geol Soc Am Bull 118:3-22.

Klein HP (1978) The Viking biological experiments on Mars. Icarus 34:666-674.

Kminek G, Conley C, Allen CC, et al. (2014) Report of the workshop for life detection in samples from Mars. Life Sci Space Res 2:1-5.

Knoll AH and Barghoorn ES (1974) Ambient pyrite in Precambrian chert: new evidence and a theory. Proc Natl Acad Sci U S A 71:2329-2331.

Knoll AH and Golubic S (1979) Anatomy and taphonomy of a Precambrian algal stromatolite. Precambrian Res 10: $115-151$.

Köhler I and Heubeck C (2019) Microbial-mat-associated tephra of the Archean Moodies Group, Barberton Greenstone Belt (BGB), South Africa: resemblance to potential biostructures and ecological implications. South Afr J Geol 122: 221-236.

Konhauser KO, Jones B, Phoenix VR, et al. (2004) The microbial role in hot spring silicification. Ambio 33:552-558.

Lachenbruch PA and Goldstein M (1979) Discriminant analysis. Biometrics 35:18.

Lepot K, Addad A, Knoll AH, et al. (2017) Iron minerals within specific microfossil morphospecies of the $1.88 \mathrm{Ga}$ Gunflint Formation. Nat Commun 8:14890.

Lollar BS, Westgate TD, Ward JA, et al. (2002) Abiogenic formation of alkanes in the Earth's crust as a minor source for global hydrocarbon reservoirs. Nature 416:3.

Lowe DR (1994) Abiological origin of described stromatolites older than 3.2 Ga. Geology 22:387.

Marshall CP, Emry JR, and Marshall AO (2011) Haematite pseudomicrofossils present in the 3. 5-billion-year-old apex chert. Nat Geosci 4:240-243.

Martel J, Young D, Peng H-H, et al. (2012) Biomimetic properties of minerals and the search for life in the martian meteorite ALH84001. Annu Rev Earth Planet Sci 40:167-193.

McCollom TM (2013) Laboratory simulations of abiotic hydrocarbon formation in Earth's deep subsurface. Rev Miner Geochem 75:467-494.

McCollom TM and Seewald JS (2006) Carbon isotope composition of organic compounds produced by abiotic synthesis under hydrothermal conditions. Earth Planet Sci Lett 243:74-84.

McKay DS, Gibson EK, Thomas-Keprta KL, et al. (1996) Search for past life on Mars: possible relict biogenic activity in martian meteorite ALH84001. Science 273:924-930.

McLachlan GJ (2004) Discriminant Analysis and Statistical Pattern Recognition. Wiley Series in Probability and Statistics, Wiley, Hoboken, NJ.

McLoughlin N, Staudigel H, Furnes H, et al. (2010) Mechanisms of microtunneling in rock substrates: distinguishing endolithic biosignatures from abiotic microtunnels: mechanisms of microtunneling in rock substrates. Geobiology 8:245-255.

McLoughlin N, Wilson LA, and Brasier MD (2008) Growth of synthetic stromatolites and wrinkle structures in the absence of microbes-implications for the early fossil record. Geobiology 6:95-105.

McMahon S. (2019) Earth's earliest and deepest purported fossils may be iron-mineralized chemical gardens. Proc $R$ Soc B Biol Sci 286:20192410.

Ménez B, Pisapia C, Andreani M, et al. (2018) Abiotic synthesis of amino acids in the recesses of the oceanic lithosphere. Nature 564:59-63.

Milesi V, Guyot F, Brunet F, et al. (2015) Formation of $\mathrm{CO}_{2}, \mathrm{H}_{2}$ and condensed carbon from siderite dissolution in the 200 $300^{\circ} \mathrm{C}$ range and at $50 \mathrm{MPa}$. Geochim Cosmochim Acta 154: 201-211.

Muscente AD, Czaja AD, Tuggle J, et al. (2018) Manganese oxides resembling microbial fabrics and their implications for recognizing inorganically preserved microfossils. Astrobiology 18:249-258.

Neveu M, Hays LE, Voytek MA, et al. (2018) The ladder of life detection. Astrobiology 18:1375-1402.

Noffke N, Eriksson KA, Hazen RM, et al. (2006) A new window into early Archean life: microbial mats in earth's oldest siliciclastic tidal deposits (3.2 Ga Moodies Group, South Africa). Geology 34:253.

Nutman AP, Bennett VC, Friend CRL, et al. (2016) Rapid emergence of life shown by discovery of 3,700-million-yearold microbial structures. Nature 537:535-538.

Oehler JH (1976) Experimental studies in Precambrian paleontology: structural and chemical changes in blue-green algae during simulated fossilization in synthetic chert. Bull Geol Soc Am 87:117-129.

Oyama VI, Berdahl BJ, Carle GC, et al. (1976) The search for life on Mars: Viking 1976 gas changes as indicators of biological activity. Origins Life 7:313-333.

Phillips J (1931) The biotic community. J Ecol 19:1.

Picard A, Schmid G, Quaroni L, et al. (2015) Experimental diagenesis of organo-mineral structures formed by microaerophilic Fe(II)-oxidizing bacteria. Nat Commun 6:1-8.

Prosser JI, Bohannan BJM, Curtis TP, et al. (2007) The role of ecological theory in microbial ecology. Nat Rev Microbiol 5: 384-392.

Rasmussen B (2000) Filamentous microfossils in a 3,235million-year-old volcanogenic massive sulphide deposit. Nature 405:4.

Rasmussen B, Fletcher IR, Brocks JJ, et al. (2008) Reassessing the first appearance of eukaryotes and cyanobacteria. Nature 455:1101-1104.

Raup DM (1967) Geometric analysis of shell coiling: coiling in ammonoids. J Paleontol 41:43-65.

Rouillard J, García-Ruiz J-M, Gong J, et al. (2018) A morphogram for silica-witherite biomorphs and its application to microfossil identification in the early Earth rock record. Geobiology 16:279-296.

Rouillard J, García-Ruiz JM, Kah L, et al. (2019) Identifying microbial life in rocks: insights from population morphometry. Geobiology 18:282-305.

Santelli CM, Banerjee N, Bach W, et al. (2010) Tapping the subsurface ocean crust biosphere: low biomass and drillingrelated contamination calls for improved quality controls. Geomicrobiol J 27:158-169.

Schopf JW (1983) Earth's Earliest Biosphere: Its Origin and Evolution, Princeton University Press, Princeton, NJ. 
Schopf JW (1993) Microfossils of the early Archean apex chert: new evidence of the antiquity of life. Science 260:640-646.

Schopf JW, Kitajima K, Spicuzza MJ, et al. (2018) SIMS analyses of the oldest known assemblage of microfossils document their taxon-correlated carbon isotope compositions. Proc Natl Acad Sci U S A 115:53-58.

Schopf JW and Kudryavtsev AB (2009) Confocal laser scanning microscopy and Raman imagery of ancient microscopic fossils. Precambrian Res 173:39-49.

Schopf JW and Kudryavtsev AB (2012) Biogenicity of Earth's earliest fossils: a resolution of the controversy. Gondwana Res 22:761-771.

Schopf JW, Kudryavtsev AB, Agresti DG, et al. (2002) Laser-Raman imagery of Earth's earliest fossils. Nature 416:73-76.

Schopf JW, Kudryavtsev AB, Sugitani K, et al. (2010) Precambrian microbe-like pseudofossils: a promising solution to the problem. Precambrian Res 179:191-205.

Schuler CG, Havig JR, Hamilton TL (2017) Hot spring microbial community composition, morphology, and carbon fixation: implications for interpreting the ancient rock record. Front Earth Sci 5:97.

Schumacher D and Søgaard-Andersen L (2017) Regulation of cell polarity in motility and cell division in Myxococcus xanthus. Annu Rev Microbiol 71:61-78.

Schwieterman EW, Kiang NY, Parenteau MN, et al. (2018) Exoplanet biosignatures: a review of remotely detectable signs of life. Astrobiology 18:663-708.

Seager S and Bains W (2015) The search for signs of life on exoplanets at the interface of chemistry and planetary science. Sci Adv 1:e1500047.

Sugitani K, Grey K, Allwood A, et al. (2007) Diverse microstructures from Archaean chert from the Mount Goldsworthy-Mount Grant area, Pilbara Craton, Western Australia: microfossils, dubiofossils, or pseudofossils? Precambrian Res 158:228-262.

Sugitani K, Grey K, Nagaoka T, et al. (2009) Taxonomy and biogenicity of Archaean spheroidal microfossils (ca. 3.0 $\mathrm{Ga}$ ) from the Mount Goldsworthy-Mount Grant area in the northeastern Pilbara Craton, Western Australia. Precambrian Res 173:50-59.

Sugitani K, Nagaoka T, Mimura K, et al. (2006) Discovery of possible microfossils from c. 3. 4 Ga Strelley Pool Chert, Kelly Group, Pilbara Craton: evidence for antiquity of life and biotic diversity? In Geoscience Research Abstracts. Presented at the European Geosciences Union, Vienna, 2006.

Sugitani K, Mimura K, Nagaoka T, et al. (2013) Microfossil assemblage from the 3400 Ma Strelley Pool Formation in the Pilbara Craton, Western Australia: results form a new locality. Precambrian Res 226:59-74.

Sugitani K, Kohama T, Mimura K, et al. (2018) Speciation of paleoarchean life demonstrated by analysis of the morphological variation of lenticular microfossils from the Pilbara Craton, Australia. Astrobiology 18:1057-1070.

Tice MM and Lowe DR (2004) Photosynthetic microbial mats in the 3,416-Myr-old ocean. Nature 431:549-552.

Ueno Y, Isozaki Y, Yurimoto H, et al. (2001) Carbon isotopic signatures of individual archean microfossils. Int Geol Rev 43:196-212.

van Zuilen MA (2018) Proposed early signs of life not set in stone. Nature 563:190-191. van Zuilen MA, Lepland A, and Arrhenius G (2002) Reassessing the evidence for the earliest traces of life. Nature 418: 627-630.

van Zuilen MA, Chaussidon M, Rollion-bard C, et al. (2007) Carbonaceous cherts of the Barberton Greenstone belt, South Africa: isotopic, chemical and structural characteristics of individual microstructures. Geochim Cosmochim Acta 71:655-669.

Wacey D, Saunders M, Kong C, et al. (2015) 3.46 Ga Apex chert "microfossils" reinterpreted as mineral artefacts produced during phyllosilicate exfoliation. Gondwana Res 36: 296-313.

Wang JD and Levin PA (2009) Metabolism, cell growth and the bacterial cell cycle. Nat Rev Microbiol 7:822-827.

Ward LM, Idei A, Terajima S, et al. (2017) Microbial diversity and iron oxidation at Okuoku-hachikurou Onsen, a Japanese hot spring analog of Precambrian iron formations. Geobiology 15:817-835.

Warming E (1895) Oecology of Plants: An Introduction to the Study of Plant Communities, Oxford University Press, London, United Kingdom.

Westall F (1999) The nature of fossil bacteria: a guide to the search for extraterrestrial life. J Geophys Res Planets 104: 16437-16451.

Westall F and Folk RL (2003) Exogenous carbonaceous microstructures in Early Archaean cherts and BIFs from the Isua Greenstone Belt: implications for the search for life in ancient rocks. Precambrian Res 126:313-330.

Westall F, Foucher F, Bost N, et al. (2015) Biosignatures on Mars: what, where, and how? Implications for the search for martian life. Astrobiology 15:998-1029.

Westfall CS and Levin PA. (2017) Bacterial cell size: multifactorial and multifaceted. Annu Rev Microbiol 71:499-517.

White G (1836) The Natural History of Selborne-with Observations on Various Parts of Nature and the Naturalist's Calendar, Whittaker and Company, London, United Kingdom.

Wolf ET and Toon OB (2010) Fractal organic hazes provided an ultraviolet shield for early Earth. Science 328:1266-1268.

Young KD (2010) Bacterial shape: two-dimensional questions and possibilities. Anпu Rev Microbiol 64:223-240.

Address correspondence to: Joti Rouillard Laboratario de Estudios Cristalograficos Instituto Andaluz de Ciencias de la Tierra CSIC-Universidad de Granada Av. de las Palmeras 4 Armilla 18100 Spain

E-mail: joti.rouillard@gmail.com

Submitted 19 April 2020

Accepted 7 September 2020

Associate Editor: Michael C. Storrie-Lombardi

\section{Abbreviations Used}

MISS $=$ microbially influenced sedimentary structures $\mathrm{SD}=$ standard deviation 